\title{
A DYNAMIC GAME OF R AND D: PATENT PROTECTION AND
} COMPETITIVE BEHAVIOR ${ }^{1}$

Replaces California Institute of Technology Social Science Working Paper 289
JENNIFER F. REINGANUM

A theory of dynamic optimal resource allocation to $\mathrm{R}$ and $\mathrm{D}$ in an $n$-firm industry is developed using differential games. This technique represents a synthesis of the analytic methods previously applied to the problem: static game theory and optimal control. The use of particular functional forms allows the computation and detailed discussion of the Nash equilibrium in investment rules.

\section{INTRODUCTION}

THIS PAPER ADDRESSES THE PROBLEM of resource allocation to research and development. Among the important issues that a firm engaging in $\mathrm{R}$ and $\mathrm{D}$ must evaluate are: uncertainty regarding the feasibility and profitability of a particular innovation; the possibility of a protracted development period; the possibility that a rival firm may innovate first, capturing either a patent or a significant share of the new market; the possibility that a rival firm may imitate the innovation and appropriate some of the profits in the new market.

In what follows, we will develop a theory of optimal resource allocation to research and development which incorporates the aspects of $R$ and $D$ enumerated above. We will use a dynamic game theoretic analysis, determining the Nash equilibrium strategies for $n$ identical firms. The availability of perfect patent protection is shown to accelerate development of the innovation, and the effect of increasing rivalry is addressed. The impact of increasing rivalry on Nash equilibrium investment in $\mathrm{R}$ and $\mathrm{D}$ depends upon the degree of appropriability of rewards. If patent protection is perfect, then increasing the number of Nash rivals results in increased $\mathrm{R}$ and $\mathrm{D}$ effort. However, when imitation is rewarded, the opposite may be true. Finally, some notions of competitive and perfectly competitive equilibrium are examined in the context of the model developed below.

\section{BACKGROUND}

The term "research and development with rivalry" is used to describe the situation in which firms compete for the profits generated by a particular innovation. More specifically, we will focus on situations in which $n \geqq 2$ firms are rivals in developing a new process or device. This rivalry may be enhanced or diminished by the number of rivals and by the availability of patent protection.

As a result of uncertainty regarding the timing of successful innovation, firms must make contingency plans for investing in research and development. Since a firm can be pre-empted at any time by a rival firm, a reasonable model of

'This paper is based on sections of my doctoral dissertation. I would like to thank Morton I. Kamien, Nancy L. Schwartz, and John Roberts for their comments and encouragement. 
induced technical change must allow firms to adjust their expenditures as a result of the temporal resolution of market uncertainty.

Basically two alternative approaches have been used to analyze $\mathrm{R}$ and $\mathrm{D}$ with rivalry: (i) a game theoretic analysis (e.g., Scherer [11], Loury [9], Lee and Wilde [8], Dasgupta and Stiglitz $[2,3])$, which is static in the sense that each firm chooses its investment strategy from a subset of $R^{+}$; (ii) a decision theoretic analysis by Kamien and Schwartz [5, 6, 7], which is dynamic in the sense that each firm chooses a function, a time path of spending on $\mathrm{R}$ and $\mathrm{D}$.

The model developed in this paper combines the two approaches described above. Rivals will be modeled as strategic agents, as in the game theoretic analysis. In addition, they will be assumed to choose strategies which may be expressed as functions of time, as in the decision theoretic analysis.

We here make the distinction between "knowledge" and "information" to be retained throughout; "knowledge" will always be used to indicate facts relevant to the firms' perfection of the innovation. That is, what they know about their research project is knowledge. What they know about each other is "information." The structure of payoffs, probability distributions, and strategy spaces constitutes information. Hence the phrase "complete information" implies that each player knows all payoff functions, probability distributions, and strategy spaces. The phrase "private knowledge" indicates that rival firms do not know the particular facts and findings of a given firm's research. Throughout this paper we will assume private knowledge. The case where research findings are a pure public good is examined in Reinganum [10].

\section{THE MODEL}

Assume that there are a number of firms engaged in a race for a particular technological breakthrough-an invention or process.

Definition 1: We say that player $i$ succeeds if $i$ perfects the device or process.

DEFINITION 2: We say that player $i$ wins if $i$ succeeds before any rival does.

If firm $i$ wins the race, then $i$ receives a payoff for being the innovator. If firm $i$ succeeds, but is not the first to succeed, then $i$ receives an imitator's payoff. If firm $i$ fails to succeed, then it gains nothing. In any case, $i$ forfeits its research investment to date.

Each player is assumed to accumulate knowledge relevant to the innovation by expending resources on research activity or knowledge acquisition. The knowledge acquisition process is assumed to be deterministic. Successful innovation, however, is assumed not deterministic. Rather, the date of successful completion of the project is a random variable which is influenced, but not determined, by firm investment in knowledge acquisition.

\section{Notation and Definitions}

This paper follows the notation of Reinganum [10].

N1. $n$ is the number of firms, indexed by $i ; n$ is assumed to be at least 2 .

N2. $T$ is the calendar date of Doomsday. Alternatively, $T$ may be regarded as a time at which the firms abandon the project entirely if they haven't yet perfected the innovation. That is, if a firm fails to succeed by $T$, it becomes convinced that the innovation is infeasible.

N3. $r$ is the discount rate common to all players.

N4. $P_{L}(t)$ is the present value of the innovation to the innovator or "leader" who wins at $t$. For convenience we will assume that this present value is the constant $P_{L}$, independent of the date of success.

N5. $P_{F}(t)$ is the present value of the reward to an imitator or "follower." Again, let us assume that $P_{F}(t)=P_{F}$, independent of the date of success. We further assume that imitation is costless and immediate, and $P_{F} \leqq P_{L}$. Because the rewards to innovation and imitation are constant in present value terms, the incentives to invest are focused upon the possibility of a rival's prior success.

N6. $t_{i}$ is the time at which firm $i$ succeeds.

N7. $\mu_{i}(t)$ is firm $i$ 's rate of knowledge acquisition at $t ; \mu_{i}(t)$ represents the additional relevant knowledge that $i$ chooses to acquire at time $t$. We further assume that $\mu_{i}(t) \in[0, B]$ where $B$ is a positive constant. That is, there is an exogenous physical limit upon the rate at which knowledge can be acquired.

N8. $e^{-r t} c_{i}\left(\mu_{i}\right)$ is the discounted cost of additional knowledge $\mu_{i}$ acquired at $t$; we assume throughout that $c_{i}\left(\mu_{i}\right)=(1 / 2)\left(\mu_{i}\right)^{2}$. In fact, the generalization to any constant elasticity cost function is straightforward and yields identical qualitative results.

N9. $z_{i}(t)$ is firm $i$ 's accumulated relevant knowledge at time $t$. Hence $\dot{z}_{i}(t)$ $=\mu_{i}(t)$, where the dot denotes the time derivative. We assume that $z_{i}(0)=0$. The bounds on $\mu_{i}(t)$ imply that $z_{i}(t)$ must lie in $Z_{i}=[0, T B]$ for all $t$ in $[0, T]$. Any base of relevant knowledge acquired earlier is reflected instead in firm $i$ 's probability of success.

N10. $F_{i}(z)$ is the probability that firm $i$ succeeds in perfecting the device or process with accumulated relevant knowledge of $z$ or less. $F_{i}(z)$ may be interpreted as an objective distribution or a subjective distribution upon which all players agree. We will specify that $F_{i}(z)=1-e^{-\lambda z}$. The use of the exponential distribution is convenient and facilitates comparison of this study with those cited earlier, which also employ the exponential distribution to represent market or technological uncertainty.

Under the subjective distribution interpretation, the exponential distribution represents an extremely "diffuse" prior since, given no success to date, each increment to knowledge is equally likely to be the one which provides success.

N1.1. Since $z_{i}(t)$ is a function of time, a probability distribution is induced over $t_{i}$. The probability that firm $i$ succeeds at or before $t$ is

$$
\operatorname{pr}\left\{t_{i} \leqq t\right\}=F_{i}\left(z_{i}(t)\right)=1-e^{-\lambda z_{i}(t)}, \quad t \in[0, T]
$$


In addition, the conditional probability that firm $i$ will succeed in the next instant, given that it has not already done so, is

$$
\operatorname{pr}\left\{t_{i} \in(t, t+d t] \mid t_{i}>t\right\}=\lambda \mu_{i}(t) d t, \quad t \in[0, T] .
$$

Notice that the instantaneous conditional probability of success depends upon the rate of knowledge acquisition selected.

N12. Vectors will be denoted by an unsubscripted letter; for instance $z=$ $\left(z_{1}, z_{2}, \ldots, z_{n}\right)$.

It is difficult to obtain results for general differential games. This formulation is meant to be suggestive of results which might be expected to hold under more general circumstances.

Because the model will be formulated in terms of both a game and a dynamic optimization problem, the following definitions are offered in an attempt to avoid confusion.

Definition 3: The strategy space for $i$ is

$$
\begin{aligned}
\mathcal{Q}_{i}=\{ & u_{i}(t, z) \mid u_{i}(t, z) \in[0, B], u_{i}(t, z) \text { is continuous in }(t, z) \\
& \text { and }\left|u_{i}(t, z)-u_{i}(t, \bar{z})\right| \leqq a(t)|z-\bar{z}| \text { for some } \\
& \text { nonnegative, integrable } a(t), \text { for all } \\
& \left.(t, z) \in[0, T] \times Z_{1} \times \cdots \times Z_{n}\right\} .
\end{aligned}
$$

A pure strategy for $i$ is an element of $\mathscr{Q}_{i}$.

Thus a pure strategy for $i$ is a function of both time and the current levels of knowledge. This type of strategy is referred to as a closed-loop strategy.

Definition 4: A control function for $i \mu_{i}(t)$ is a continuous function taking values in the control space $U_{i}=[0, B]$ for all $t$ in $[0, T]$.

Definition 5: The kinematic equations are a system of equations which govern the evolution over time of the state variables $z_{1}, z_{2}, \ldots, z_{n}$. The kinematic equations depend on the players' strategies:

$$
\dot{z}_{i}=g_{i}(t, z, u) \quad(i=1,2, \ldots, n) .
$$

For any pure strategy $n$-tuple $\left(u_{1}(t, z), \ldots, u_{n}(t, z)\right)$, the kinematic equations become a system of ordinary differential equations:

$$
\dot{z}_{i}=g_{i}(t, z, u(t, z))
$$

$$
(i=1,2, \ldots, n)
$$

Definition 6: A solution to the system (1) through given initial conditions is a differentiable curve $z(t)=\left(z_{1}(t), \ldots, z_{n}(t)\right)$ defined for all $t$ in $[0, T]$ such that $\dot{z}_{i}(t)=g_{i}(t, z(t)), i=1,2, \ldots, n$, and such that $z(t)$ satisfies the given initial conditions.
In our case $g_{i}(t, z)=u_{i}(t, z)$ where $u_{i}(t, z)$ is any pure strategy for $i$. By theorems from differential equations (see, e.g., Friedman [4, pp. 4-5]), the assumptions of continuity, boundedness, and the Lipschitz conditions in $z$ are sufficient to guarantee the existence of a unique solution to the system (1) through $z(0)=0$.

Definition 7: The control functions associated with a particular strategy $n$ tuple $\left(u_{1}(t, z), \ldots, u_{n}(t, z)\right)$ are

$$
\left(\mu_{1}(t), \mu_{2}(t), \ldots, \mu_{n}(t)\right)=\left(u_{1}(t, z(t)), u_{2}(t, z(t)), \ldots, u_{n}(t, z(t))\right),
$$

where $z(t)$ is the solution of the system $\dot{z}=u(t, z)$ through $z(0)=0$.

In order to define the game, we must specify the players' strategy spaces $\mathscr{Q}_{i}$ and their payoffs $J^{i}\left(u_{1}, \ldots, u_{n}\right)$, for $i=1,2, \ldots, n$. We have defined $\mathcal{Q}_{i}$ in Definition 1 above.

The payoff to player $i$ consists of three terms: firm $i$ receives the amount $P_{L}$ (in present value terms) at $t$ if no firm has yet succeeded and if $i$ succeeds at $t$ (given that it has not yet succeeded). That is, firm $i$ receives $P_{L}$ at $t$ with probability $\exp \left\{-\lambda \sum z_{k}(t)\right\} \lambda \mu_{i}(t)$. Furthermore, since imitation is costless and immediate, firm $i$ receives an imitator's payoff $P_{F}$ at $t$ if no firm has succeeded by $t$ and if any other firm succeeds at $t$ (given that it has not already succeeded). That is, firm $i$ receives present value $P_{F}$ at $t$ with probability $\exp \left\{-\lambda \sum z_{k}(t)\right\}$. $\left[\sum_{j \neq i} \lambda \mu_{j}(t)\right]$. However, development costs of $(1 / 2)\left(\mu_{i}(t)\right)^{2}$ accompany research activity so long as no player has succeeded. That is, firm $i$ incurs costs of $(1 / 2)\left(\mu_{i}(t)\right)^{2}$ with probability $\exp \left\{-\lambda \sum z_{k}(t)\right\}$. Combining these terms and discounting the flow costs at rate $r$, we can write firm $i$ 's payoff for any strategy $n$-tuple $\left(u_{1}, \ldots, u_{n}\right)$ as follows:

$$
\begin{aligned}
J^{i}\left(u_{1}, \ldots, u_{n}\right)=\int_{0}^{T} & \left\{P_{L} e^{-\lambda \sum z_{k}(t)} \lambda \mu_{i}(t)+P_{F} e^{-\lambda \sum z_{k}(t)} \sum_{j \neq i} \lambda \mu_{j}(t)\right. \\
& \left.-e^{-r t} e^{-\lambda \sum z_{k}(t)}(1 / 2)\left(\mu_{i}(t)\right)^{2}\right\} d t
\end{aligned}
$$

where $\mu_{i}(t)=u_{i}(t, z(t))$ and $z(t)$ solves $\dot{z}=u(t, z)$ with $z(0)=0$. We use the abbreviated $\left(u_{1}, \ldots, u_{n}\right)$ to represent the entire strategy $n$-tuple $\left(u_{1}(t, z)\right.$, $\left.\ldots, u_{n}(t, z)\right)$ over all of $[0, T] \times Z_{1} \times \cdots \times Z_{n}$.

It will prove more convenient to integrate the first term of the payoff by parts and deal with the resulting equivalent payoffs:

$$
\begin{aligned}
J^{i}\left(u_{1}, \ldots, u_{n}\right)= & \int_{0}^{T}\left\{\left[P_{L}\left(1-e^{-\lambda z_{i}(t)}\right)+P_{F} e^{-\lambda z_{i}(t)}\right] \exp \left\{-\lambda \sum_{k \neq i} z_{k}(t)\right\}\right. \\
& \left.\times \sum_{j \neq i} \lambda \mu_{j}(t)-e^{-r t} e^{-\lambda \sum z_{k}(t)}(1 / 2)\left(\mu_{i}(t)\right)^{2}\right\} d t \\
& +P_{L}\left(1-e^{-\lambda z_{i}(T)}\right) \exp \left\{-\lambda \sum_{k \neq i} z_{k}(T)\right\},
\end{aligned}
$$

for $i=1,2, \ldots, n$ 


\section{THE NASH EQUILIBRIUM}

In order to propose a method of solution, we must first decide upon the players' behavioral attitudes. We assume that each firm maximizes its own payoff, taking its rivals' strategies as given. This assumption results in the Nash equilibrium concept.

Definition 8: A strategy $n$-tuple $\left(u_{1}^{*}, u_{2}^{*}, \ldots, u_{n}^{*}\right)$ is a Nash equilibrium if

$$
u_{i}^{*}(t, z) \in \mathcal{Q}_{i}
$$

$(i=1,2, \ldots, n)$

and

$$
J^{i}\left(u_{1}^{*}, \ldots, u_{i-1}^{*}, u_{i}^{*}, u_{i+1}^{*}, \ldots, u_{n}^{*}\right) \geqq J^{i}\left(u_{1}^{*}, \ldots, u_{i-1}^{*}, u_{i}, u_{i+1}^{*}, \ldots, u_{n}^{*}\right)
$$

for all $u_{i} \in \mathcal{Q}_{i}, i=1,2, \ldots, n$.

Define the value functions

$$
\begin{aligned}
V^{i}(s, y)= & \int_{s}^{T}\left\{\left[P_{L}\left(1-e^{-\lambda z_{i}(t)}\right)+P_{F} e^{-\lambda z_{i}(t)}\right] \exp \left\{-\lambda \sum_{k \neq i} z_{k}(t)\right\}\right. \\
& \left.\times \sum_{j \neq i} \lambda \mu_{j}^{*}-e^{-r t} e^{-\lambda \sum z_{k}(t)}(1 / 2)\left(\mu_{i}^{*}(t)\right)^{2}\right\} d t \\
& +P_{L}\left(1-e^{-\lambda z_{i}(T)}\right) \exp \left\{-\lambda \sum_{k \neq i} z_{k}(T)\right\}
\end{aligned}
$$

where $y=z(s)$.

Then from Theorem 8.2 .2 (Friedman [4]), we know that $\left(u_{1}^{*}, \ldots, u_{n}^{*}\right)$ must satisfy a system of Bellman equations: for $i=1,2, \ldots, n$,

$$
\begin{aligned}
V_{t}^{i}(t, z)+\max _{u_{i}(t, z) \in[0, B]}[ & V_{z_{i}}^{i}(t, z) u_{i}(t, z)+\sum_{j \neq i} V_{z_{j}}^{i}(t, z) u_{j}^{*}(t, z) \\
& +\left[P_{L}\left(1-e^{-\lambda z_{i}}\right)+P_{F} e^{-\lambda z_{i}}\right] \exp \left\{-\lambda \sum_{k \neq t} z_{k}\right\} \\
& \times \sum_{j \neq i} \lambda u_{j}^{*}(t, z)-e^{-r t} \exp \left\{-\lambda \sum z_{k}\right\} \\
& \left.\times(1 / 2)\left(u_{i}(t, z)\right)^{2}\right]=0 \quad(i=1,2, \ldots, n) .
\end{aligned}
$$

Then by the definition of $V^{i}(s, y)$, for all $z$

$$
V^{i}(T, z)=P_{L} \exp \left\{-\lambda \sum_{k \neq i} z_{k}\right\}-P_{L} \exp \left\{-\lambda \sum z_{k}\right\}
$$

The candidates for a Nash equilibrium can be obtained by performing the indicated maximization. Since the left hand side of equation (2) is strictly concave in $u_{i}$, the necessary conditions are also sufficient for maximization:

$$
\begin{aligned}
& V_{z_{i}}^{i}(t, z)-e^{-r t} e^{-\lambda \sum z_{k}} u_{i}(t, z) \\
& \left\{\begin{array}{lll}
>0 & \text { for all } & u_{i}(t, z) \in[0, B] \quad \text { implies } u_{i}^{*}(t, z)=B, \\
=0 & \text { implies } & u_{i}^{*}(t, z)=V_{z_{i}}^{i}(t, z) e^{r t} e^{\lambda \sum z_{k}}, \\
<0 & \text { for all } & u_{i}(t, z) \in[0, B] \text { implies } u_{i}^{*}(t, z)=0,
\end{array}\right.
\end{aligned}
$$

for $i=1,2, \ldots, n$.

The first-order conditions indicate that if the optimal rate of knowledge acquisition is to be within the interval $(0, B)$, then the discounted expected marginal cost rate must be exactly balanced by the incremental expected profit to the firm generated by that rate of expenditure. If such a balance cannot be struck, then the appropriate boundary conditions determine the optimal rate of knowledge acquisition.

It is difficult to describe the Nash equilibrium strategies without solving explicitly for the value functions.

Suppose we concentrate on interior solutions, where $u_{i}^{*}(t, z) \in(0, B)$ for all $(t, z) \in[0, T] \times Z_{1} \times \cdots \times Z_{n}$. If we substitute the expressions

$$
u_{i}^{0}=V_{z_{i}}^{i} e^{r t} e^{\lambda \sum z_{k}}
$$

back into the Bellman system (2)-(3) and if we can solve the resulting system of Hamilton-Jacobi equations for the value functions $V^{1}(t, z)$ through $V^{n}(t, z)$, then we can determine the Nash equilibrium (candidate) strategies via the substitution

$$
u_{i}^{*}(t, z)=u_{i}^{0}\left(t, z, V_{z}(t, z)\right)
$$

Substituting the expressions $\left(u_{1}^{0}, \ldots, u_{n}^{0}\right)$ into system (2) and simplifying yields

$$
\begin{aligned}
V_{t}^{i}+ & (1 / 2)\left(V_{z_{i}}^{i}\right)^{2} e^{r t} e^{\lambda \sum z_{k}}+\sum_{j \neq i} V_{z_{j}}^{i} V_{z_{j}}^{j} e^{r t} e^{\lambda \sum z_{k}} \\
& +\left[P_{L}\left(e^{\lambda z_{i}}-1\right)+P_{F}\right] \lambda \sum_{j \neq i} V_{z_{j}}^{j} e^{r t}=0
\end{aligned}
$$

for $i=1,2, \ldots, n$, with terminal conditions (3).

This suggests a solution of the form

$$
V^{i}(t, z)=b(t) \exp \left\{-\lambda \sum z_{k}\right\}+a(t) \exp \left\{-\lambda \sum_{k \neq i} z_{k}\right\}
$$


$i=1,2, \ldots, n$. Substituting the appropriate partial derivatives into (4) and collecting terms gives

$$
\begin{aligned}
\exp \{ & \left.-\lambda \sum z_{k}\right\}\left[\dot{b}(t)+(b(t))^{2} \lambda^{2} e^{r t}(2 n-1) / 2\right. \\
& \left.+b(t)(n-1)\left(P_{L}-P_{F}\right) \lambda^{2} e^{r t}\right]+\exp \left\{-\lambda \sum_{k \neq i} z_{k}\right\} \\
& \times\left[\dot{a}(t)+a(t) b(t)(n-1) \lambda^{2} e^{r t}-\lambda^{2} b(t) P_{L}(n-1) e^{r t}\right]=0
\end{aligned}
$$

Since $\exp \left\{-\lambda \sum z_{k}\right\}$ and $\exp \left\{-\lambda \sum_{k \neq i} z_{k}\right\}$ are always positive, the coefficient functions $b(t)$ and $a(t)$ must solve the ordinary differential equations and boundary conditions

$$
\dot{b}(t)+(b(t))^{2} \lambda^{2} e^{r t}(2 n-1) / 2+b(t)(n-1)\left(P_{L}-P_{F}\right) \lambda^{2} e^{r t}=0,
$$

$$
\begin{aligned}
& b(T)=-P_{L} . \\
& \dot{a}(t)+a(t) b(t)(n-1) \lambda^{2} e^{r t}-b(t) P_{L}(n-1) \lambda^{2} e^{r t}=0, \\
& a(T)=P_{L} .
\end{aligned}
$$

Equation (6) can be reduced to a linear ordinary differential equation via the substitution $g(t)=-1 / b(t)$, and therefore has the unique solution through $b(T)=-P_{L}$,

$$
b\left(t ;-P_{L}\right)=\frac{-2(n-1)\left(P_{L}-P_{F}\right) P_{L}}{(2 n-1) P_{L}-\left[P_{L}+2(n-1) P_{F}\right] \exp \{m(t)\}}
$$

where $m(t)=\left(P_{L}-P_{F}\right)(n-1) \lambda^{2}\left(e^{r t}-e^{r T}\right) / r$. Notice that if $P_{L} \geqq P_{F}$, then $b\left(t ;-P_{L}\right)<0$ for all $t \leqq T$.

The second coefficient must solve (7). The general solution to this nonhomogenous linear ODE is $a\left(t ; P_{L}\right)=P_{L}$.

We can now write the value functions for $n$ identical Nash rivals:

$$
\begin{aligned}
V^{i}\left(t, z ; P_{L}, P_{F}\right)= & P_{L} \exp \left\{-\lambda \sum_{k \neq i} z_{k}\right\} \\
& -\frac{2\left(P_{L}-P_{F}\right)(n-1) P_{L} e^{-\lambda \sum z_{k}}}{(2 n-1) P_{L}-\left[P_{L}+2(n-1) P_{F}\right] \exp \{m(t)\}}
\end{aligned}
$$

We can now state the following proposition.
Proposition 1: Suppose $B \geqq P_{L} \lambda e^{r T}$. When imitation is rewarded by $P_{F}\left(<P_{L}\right)$,

(a) $\quad u_{i}^{*}\left(t, z ; P_{L}, P_{F}\right)=\frac{2 \lambda P_{L}\left(P_{L}-P_{F}\right)(n-1) e^{r t}}{(2 n-1) P_{L}-\left[P_{L}+2(n-1) P_{F}\right] \exp \{m(t)\}}$,

$i=1,2, \ldots, n$, is the unique Nash equilibrium in $\mathscr{q}_{1} \times \cdots \times \mathcal{Q}_{n}$.

$$
J^{i}\left(u_{1}^{*}, \ldots, u_{n}^{*}\right)=P_{L}-\frac{2\left(P_{L}-P_{F}\right) P_{L}(n-1)}{(2 n-1) P_{L}-\left[P_{L}+2(n-1) P_{F}\right] \exp \{m(t)\}},
$$$$
i=1,2, \ldots, n \text {. }
$$

Proof (a): Clearly $\left(u_{1}^{*}, \ldots, u_{n}^{*}\right) \in \mathcal{Q}_{1} \times \cdots \times \mathcal{Q}_{n}$. We presented a continuously differentiable solution $\left(V^{1}\left(t, z ; P_{L}, P_{F}\right), \ldots, V^{n}\left(t, z ; P_{L}, P_{F}\right)\right)$ to the system (3)-(4). By construction, $V^{i}\left(t, z ; P_{L}, P_{F}\right)$ and $u_{i}^{*}(t, z)=V_{z_{i}}^{i}\left(t, z ; P_{L}, P_{F}\right) e^{r t} e^{\lambda \sum z_{k}}$ satisfy the hypotheses of the sufficiency Theorem 1 (Stalford and Leitmann [12]). Therefore $\left(u_{1}^{*}\left(t, z ; P_{L}, P_{F}\right), \ldots, u_{n}^{*}\left(t, z ; P_{L}, P_{F}\right)\right)$ as above is a Nash equilibrium.

Since the left-hand side of system (4) is of class $C^{\infty}$ in $\left(t, z, V_{z}\right)$ and since the terminal functions in (3) are of class $C^{\infty}$ in $z$, by Theorem 18.1 (Bernstein [1]), there is only one solution to (3)-(4). Thus $\left(u_{1}^{*}\left(t, z ; P_{L}, P_{F}\right), \ldots, u_{n}^{*}\left(t, z ; P_{L}, P_{F}\right)\right)$ as defined above is the unique Nash equilibrium in $\mathscr{U}_{1} \times \cdots \times \mathcal{U}_{n}$.

Proof (b): The value of profits obtained by playing the Nash equilibrium strategies are $J^{i}\left(u_{1}^{*}, \ldots, u_{n}^{*}\right)=V^{i}\left(0,0 ; P_{L}, P_{F}\right)$ for $i=1,2, \ldots, n$.

Q.E.D.

The case of perfect patent protection is of especial interest, since it is by far the case most commonly examined. Denote the patent value by $P$. Then $P_{L}=P$ while $P_{F}=0$.

COROLlary 1: The Nash equilibrium strategies under perfect patent protection are

$$
u_{i}^{*}(t, z ; P, 0)=\frac{2 \lambda(n-1) P e^{r t}}{2 n-1-\exp \left\{P \lambda^{2}(n-1)\left(e^{r t}-e^{r T}\right) / r\right\}}
$$

$$
(i=1,2, \ldots, n) \text {. }
$$

Due to the "memorylessness" property of the exponential distribution, the control functions associated with the Nash equilibrium strategies are the strategies themselves. That is, the Nash equilibrium strategies are functions of time only. This result is of some interest on technical grounds. Since Nash equilibria 
in closed-loop and open-loop strategies do not coincide in general, this mode represents a class of such solvable games. On the other hand, this result will not generalize to alternative distribution functions.

The control function $\mu_{i}^{*}(t)$ is the additional knowledge acquired by firm $i$ at $t$. The cost of this knowledge is $(1 / 2)\left(\mu_{i}^{*}(t)\right)^{2}$. To determine whether firms invest at a higher or lower rate as time progresses, we need to determine the sign of $\dot{c}_{i}\left(\mu_{i}^{*}(t)\right)$.

Proposition 2: $\dot{c}_{i}\left(\mu_{i}^{*}(t)\right)>0$ for all $t \leqq T$.

Proof: Differentiate and collect terms.

COROLlARY 2: We can now note that the instantaneous conditional probability of success $\lambda \mu_{i}^{*}(t)$ is an increasing function of time. That is, as time passes, it becomes increasingly likely that firm $i$ will succeed in the next time increment dt, given that it has not yet succeeded.

The following proposition summarizes the effects of varying the payoffs to innovator and imitator.

Proposition 3: For $P_{L}>P_{F}$, (a) $\partial u_{i}^{*} / \partial P_{L}>0$ for all $t \leqq T$; that is, an increase in the payoff to the innovator stimulates firm $i$ to proceed at a faster rate; (b) $\partial u_{i}^{*} / \partial P_{F}<0$ for all $t<T$ with $\partial u_{i}^{*} / \partial P_{F}=0$ at $t=T$; that is, an increase in the payoff to imitation causes the firm to reduce its equilibrium rate of knowledge acquisition.

Proof (a): Let $m(t)=\left(P_{L}-P_{F}\right)(n-1) \lambda^{2}\left(e^{r t}-e^{r T}\right) / r$. Notice that $m(t) \leqq 0$ for all $t \leqq T$.

$$
\partial u_{i}^{*} / \partial P_{L}=\frac{2 \lambda(n-1) e^{r t} h(m)}{\left((2 n-1) P_{L}-\left[P_{L}+2(n-1) P_{F}\right] e^{m}\right)^{2}}
$$

where $h(m)=P_{L}^{2}(2 n-1)+e^{m}\left\{P_{L}^{2}(m-1)+2 P_{L} P_{F}(n-1)(m-2)+2 P_{F}^{2}(n-\right.$ 1)\}. Then $\operatorname{sgn} \partial u_{i}^{*} / \partial P_{L}=\operatorname{sgn} h(m)$. Now

$$
\begin{aligned}
& h(0)=P_{L}^{2}(2 n-1)-P_{L}^{2}-4 P_{L} P_{F}(n-1)+2 P_{F}^{2}(n-1) \\
& \quad=2(n-1)\left(P_{L}-P_{F}\right)^{2}>0 ; \quad \text { and } \\
& h^{\prime}(m)=e^{m}\left\{P_{L}^{2} m+2 P_{L} P_{F}(n-1) m+2(n-1) P_{F}\left(P_{F}-P_{L}\right)\right\}<0,
\end{aligned}
$$

for all $m \leqq 0(t \leqq T)$ and $P_{L}>P_{F}$. Thus as $m$ decreases, $h(m)$ increases from $2(n-1)\left(P_{L}-P_{F}\right)^{2}$. Therefore $h(m)>0$ for all $m \leqq 0(t \leqq T)$, and claim (a) follows.

Proof (b):

$$
\partial u_{i}^{*} / \partial P_{F}=\frac{2 P_{L}(n-1) \lambda e^{r t} y(m)}{\left((2 n-1) P_{L}-\left[P_{L}+2(n-1) P_{F}\right] e^{m}\right)^{2}}
$$

where $y(m)=P_{L} e^{m}(2 n-1-m)-(2 n-1) P_{L}-2 P_{F}(n-1) m e^{m}$. Then $\operatorname{sgn} \partial u_{i}^{*} / \partial P_{F}=\operatorname{sgn} y(m)$. Now $y(0)=0$ and

$$
y^{\prime}(m)=e^{m}\left\{2(n-1)\left(P_{L}-P_{F}\right)-\left(P_{L}+2(n-1) P_{F}\right) m\right\}>0
$$

for all $m \leqq 0$. Thus as $m$ decreases, $y(m)$ decreases from $y(0)=0$. Therefore $\partial u_{i}^{*} / \partial P_{F}<0$ for all $t<T$, while $\partial u_{i}^{*} / \partial P_{F}=0$ at $t=T$.

From this proposition, it is clear that firms will generate knowledge at a uniformly higher rate when patent protection is perfect than when protection is imperfect. Formally, we have the following corollary.

$$
\begin{aligned}
& \text { Corollary 3: For } P_{L}<P, P_{F} \geqq 0, \\
& \qquad u_{i}^{*}(t, z ; P, 0)>u_{i}^{*}\left(t, z ; P_{L}, P_{F}\right) \quad \text { for all } \quad t \leqq T .
\end{aligned}
$$

This corollary confirms our intuition that firms will expend greater effort if they can be assured of collecting the entire reward.

Consider the limiting case $P_{L}=P_{F}$. In a deterministic game, if imitation and innovation are equally rewarded, then Nash equilibrium will fail to exist, with each firm preferring to imitate rather than innovate. This is not the case in the presence of technological uncertainty. Taking the limit as $P_{L}$ decreases to $P_{F}$ (using l'Hopital's rule) yields the Nash equilibrium strategies when innovation and imitation are equally rewarded:

$$
u_{i}^{*}\left(t, z ; P_{F}, P_{F}\right)=\frac{2 P_{F} \lambda e^{r t}}{2-(2 n-1) P_{F} \lambda^{2}\left(e^{r t}-e^{r T}\right) / r} \quad(i=1, \ldots, n) .
$$

In the presence of technological uncertainty, a firm cannot simply wait for its rival to innovate, since given the rivals' strategies, there remains a positive probability that no rival will succeed within the planning horizon. If a firm wants any payoff, its optimal strategy is to pursue the payoff actively, rather than simply to wait for a rival to succeed and then reap the reward to imitation.

Further comparative statics results are summarized below. 
Proposition 4: (a) If $P_{L}>4 P_{F}(n-1) e^{-1 / 2} /\left[2 n-1-2 e^{-1 / 2}\right]$, then $\partial u_{i}^{*} / \partial \lambda$ $>0$ for all $t \leqq T$; (b) otherwise $\partial u_{i}^{*} / \partial \lambda \leqq 0$ for some $t$; however, $\partial u_{i}^{*} / \partial \lambda>0$ near $t=T$.

Proof (a): For $P_{L}>P_{F}$,

$$
\partial u_{i}^{*} / \partial \lambda=\frac{2 P_{L}(n-1)\left(P_{L}-P_{F}\right) e^{r t}\left[(2 n-1) P_{L}+\alpha e^{m}(2 m-1)\right]}{\left[(2 n-1) P_{L}-\alpha e^{m}\right]^{2}}
$$

where $\alpha=P_{L}+2(n-1) P_{F}$ and $m(t)=\left(P_{L}-P_{F}\right) \lambda^{2}\left(e^{r t}-e^{r T}\right) / r$. Let $f(m)$ $=(2 n-1) P_{L}+\alpha e^{m}(2 m-1)$. Then $\operatorname{sgn} \partial u_{i}^{*} / \partial \lambda=\operatorname{sgn} f(m)$. Now $f^{\prime}(m) \gtreqless 0$ as $m \gtreqless-1 / 2$, so $f(m)$ attains a minimum at $m=-1 / 2$. But $f(-1 / 2)>0$ under (a). Therefore $f(m)>0$ for all $m$, implying that $\partial u_{i}^{*} / \partial \lambda>0$ for all $t \leqq T$.

Proof (b): For $P_{F}<P_{L} \leqq 4 P_{F}(n-1) e^{-1 / 2} /\left[2 n-1-2 e^{-1 / 2}\right], f(-1 / 2) \leqq 0$, so $\partial u_{r}^{*} / \partial \lambda \leqq 0$ in a neighborhood of $m=-1 / 2$; but $f(0)=2(n-1)\left(P_{L}-P_{F}\right)$ $>0$, so $\partial u_{i}^{*} / \partial \lambda>0$ when $m=0(t=T)$.

Q.E.D.

For the limiting case of $P_{L}=P_{F}$,

$$
\partial u_{i}^{*} / \partial \lambda=\frac{2 P_{F} e^{r t}[2+(2 n-1) \hat{m}]}{[2-(2 n-1) \hat{m}]^{2}}
$$

where $\hat{m}(t)=P_{F} \lambda^{2}\left(e^{r t}-e^{r T}\right) / r$. Thus $\partial u_{i}^{*} / \partial \lambda \leqq 0$ for all $\hat{m} \leqq-2 /(2 n-1)$; but $\partial u_{i}^{*} / \partial \lambda>0$ at $\hat{m}=0(t=T)$.

The impact of a decrease in the mean level of knowledge required for success, $1 / \lambda$, is complicated by the possibility of imperfect patent protection. Part (a) indicates that if innovation is sufficiently highly rewarded (relative to imitation), then firms will invest at a uniformly higher rate in projects which require (on average) less knowledge. In particular, this is true for the case of perfect patent protection. However, in the extreme case where patent protection is totally ineffective, throughout most of the horizon firms would invest at a higher rate on a project requiring (on average) more knowledge.

Proposition 5: (a) $\partial u_{i}^{*} / \partial T<0$ for all $t \leqq T$; as the planning horizon increases, firms spread out their $\mathrm{R}$ and $\mathrm{D}$ effort over the extended horizon. (b) However, $\partial z_{i}^{*}(T) / \partial T>0 ;$ maximum planned knowledge accumulation $z_{i}(T)$ increases with $T$.

Proof (a): Differentiate and collect terms.

Proof (b): Find $z_{i}^{*}(T)$ using $\dot{z}_{i}=u_{i}^{*}$ and $z_{i}(0)=0$. Then differentiate and collect terms.

At this point we can remark that the infinite horizon case can be examined via a limiting argument. The Nash equilibrium strategies for the infinite horizon case are

$$
\lim _{T \rightarrow \infty} u_{i}^{*}\left(t, z ; P_{L}, P_{F}\right)=\frac{2\left(P_{L}-P_{F}\right)(n-1) \lambda e^{r t}}{2 n-1} .
$$

In this section we have been able to determine the Nash equilibrium strategies and the nature of their dependence upon most of the parameters. An exception is the discount rate $r$. The sign of $\partial u_{i}^{*} / \partial r$ is not uniform over time. Instead there may be multiple discontinuities in the functions sgn $\partial u_{i}^{*} / \partial r$.

In the following section, we examine the dependence of the Nash equilibrium strategies upon the number of rivals.

\section{IS COMPETITION CONDUCIVE TO TECHNICAL ADVANCE?}

This question has been asked-and answered differently-many times. Part of the confusion must lie with the vagueness of the term "competition." Does "competition" (or rivalry) consist of a behavioral attitude or the number of firms in the industry? The players in this paper are competitive in the sense that they are noncooperative. Yet if patent protection is perfect, while all firms compete for the patent, there will be no competitors (or imitators) once the innovator succeeds. If patent protection is imperfect, then there is competition for rewards as well as for the perfection of the innovation. That is, the firms are competitors both before and after successful innovation.

In this study, we will assume Nash behavior and "increasing competition" or "increasing rivalry" will refer to increasing the number of these rivals.

With few exceptions (Kamien and Schwartz [5, 7]), the recent literature on $R$ and $\mathrm{D}$ with rivalry focuses on the case of perfect patent protection. To facilitate comparison of the results of this study with those of others, we will temporarily restrict our analysis to the perfect patent protection case.

Although Kamien and Schwartz do not explicitly include the number of rivals in their analysis [7], because their firm possesses a subjective probability distribution function over the collectivity of its rivals' introduction date-assumed to be exponential with parameter $h$-it seems reasonable that an increase in the number of rivals would result in an increase in the subjective parameter $h$. Since $1 / h$ is the expected time until innovation occurs, it is reasonable that more firms should decrease this expression. In a recent paper, Kamien and Schwartz [7] show that, for the case of perfect patent protection and no technological uncertainty, either (i) increasing $h$ always results in a lengthening of the development period; or (ii) increasing $h$ initially decreases the choice of development period. However, after some point $h^{*}$ increased rivalry results in a lengthening of the optimal development period for an individual firm using a decision theory
analysis.

In his recent game theoretic model Loury [9] uses the exponential distribution to represent technological uncertainty. In particular, each firm $i$ has probability $1-\exp \left\{-h\left(x_{1}\right) t\right\}$ of success by time $t$. Thus firm $i$ faces a probability that a rival has succeeded by $t$ of $1-e^{-a_{i} t}$ where $a_{i}=\sum_{j \neq i} h\left(x_{j}\right), h\left(x_{j}\right)$ is the parameter 
from $j$ 's distribution and $x_{j}$ is firm $j$ 's choice of $\mathrm{R}$ and $\mathrm{D}$ expenditures. Thus for symmetric firms, $a_{i}=(n-1) h\left(x_{j}\right)$. Since $x_{j}$ is taken as given, firm $i$ regards $a_{i}$ as a parameter. Then "increasing rivalry" is taken to be an increase in $n$, the number of firms in the industry. Loury finds that, in equilibrium, an increase in $n$ unambiguously causes a decrease in $x_{i}^{*}$. However, given a certain stability condition, industry investment $n x_{i}^{*}$ increases with $n$. That is, increased rivalry results in an earlier innovation date (on average) even though individual firm investment declines.

Loury's firms choose the level of resources they wish to expend, $x_{i}$; this buys them a distribution $\tau_{i}\left(x_{i}\right)$ over the time of their actual success. Notice that the amount $x_{i}$ is a sunk cost once time passes $t=0$. That is, if a firm succeeds at $t=\epsilon>0$, then the other firms are unable to limit their losses to the expenditures to date. Thus increased rivalry has no effect on $i$ 's expected costs. However, the expected reward declines with the addition of rivals since now firm $i$ has less chance of capturing the patent. Under these circumstances it is not surprising that firms choose to invest a lower amount should additional rivals appear.

Lee and Wilde's [8] modification of Loury's model reverses this result. That is, if instead of a fixed cost of $x_{i}$, a flow cost of $x_{i}$ is paid only until some firm wins, and if the random time of success depends upon the magnitude of this flow cost, then both expected benefits and expected costs decline as the number of rivals increases. The resulting impact on equilibrium investment $x_{i}^{*}$ is positive-as the number of rivals increases, each invests at a higher rate $x_{i}^{*}$.

Since, in reality, firms can (and would) cease development if a rival preempts them (and patent protection is perfect), it seems more reasonable that both firm $i$ 's expected rewards and its expected costs will decline as rivalry increases. The model developed in this paper allows such flexibility.

Recall that when patent protection is perfect, each of $n$ identical firms plays the Nash equilibrium strategy

$$
u_{i}^{*}(t, z ; P, 0)=\frac{2 P \lambda(n-1) e^{r t}}{2 n-1-\exp \left\{P \lambda^{2}(n-1)\left(e^{r t}-e^{r T}\right) / r\right\}} .
$$

In order to determine the effect of increased rivalry (as represented by an increase in the number of firms) on individual investment, we treat $n$ as a continuous parameter and examine $\partial u_{i}^{*} / \partial n$.

Proposition 6: Suppose $n \geqq 2$. Then

$$
\partial u_{i}^{*} / \partial n>0 \text { for all } t<T \text { and } \partial u_{i}^{*} / \partial n=0 \text { at } t=T .
$$

That is, an increase in the number of Nash rivals results in an increase in each firm's individual equilibrium investment in $\mathrm{R}$ and $\mathrm{D}$. A fortiori an increase in the number of rivals tends to hasten technical advance.
Proof: Let $m(t)=P \lambda^{2}(n-1)\left(e^{r t}-e^{r T}\right) / r$. Note that $m(t)<0$ whenever $t<T$.

$$
\partial u_{i}^{*} / \partial n=\frac{2 P \lambda e^{r t}\left[1-e^{m}(1-m)\right]}{\left(2 n-1-e^{m}\right)^{2}} .
$$

Let $g(m)=1-e^{m}(1-m)$. Now $\operatorname{sgn} \partial u_{i}^{*} / \partial n=\operatorname{sgn} g(m)$. But $g(0)=0$ and $g^{\prime}(m)$ $=m e^{m}<0$ for all $t<T$. Therefore $g(m)>0$ for all $t<T$ and $g(0)=0$ at $t=T$. The proposition follows.

Q.E.D.

Although all of the models mentioned here-Kamien and Schwartz [7], Loury [9], Lee and Wilde [8], and the current model-are remarkably similar, we have arrived at a variety of conclusions regarding the impact of additional rivals on individual equilibrium investment in $\mathrm{R}$ and $\mathrm{D}$. The current model is in agreement with Lee and Wilde on this point. As discussed earlier, it is the assumption that the date of success depends upon fixed rather than flow costs which is responsible for the discrepancy between the results of this study and those of Loury. The disagreement between this study and that of Kamien and Schwartz may be attributable to the game versus decision theoretic assumption.

Therefore the answer to the question "is competition conducive to technical advance?" depends upon whether the firms' decision processes are more closely approximated by the game or decision theory analysis, and whether it is a fixed or flow cost which is most important in determining the date of successful innovation.

When patent protection is imperfect, the answer is even more ambiguous. Although Kamien and Schwartz [7] report that in this case innovation is unambiguously delayed by an increase in the number of rivals, the game model does not confirm this.

It is reasonable to suggest that the payoffs to innovation and imitation, $P_{L}$ and $P_{F}$, respectively, are determined in a game of oligopoly following successful innovation. Since the total benefits to be extracted from the market are limited, the values $P_{L}$ and $P_{F}$ will depend upon the number of rivals among whom the market is to be divided. Now since $P_{L}=P_{L}(n)$ and $P_{F}=P_{F}(n)$, to determine the effect of increasing rivalry on equilibrium production of knowledge we need to determine the sign of

$$
\frac{d u_{i}^{*}}{d n}=\frac{\partial u_{i}^{*}}{\partial n}+\frac{\partial u_{i}^{*}}{\partial P_{L}} \frac{d P_{L}}{d n}+\frac{\partial u_{i}^{*}}{\partial P_{F}} \frac{d P_{F}}{d n} .
$$

The first term may be of either sign, although it is negative for $t$ near $T$. We saw earlier that $\partial u_{i}^{*} / \partial P_{L}>0$, while $\partial u_{i}^{*} / \partial P_{F} \leqq 0$. Since both $d P_{L} / d n$ and $d P_{F} / d n$ are likely to be negative, the sign of $d u_{i}^{*} / d n$ is ambiguous and will depend upon the nature of the $n$-firm oligopoly game solution.

Thus the effect of increasing rivalry is considerably more complicated when patent protection is imperfect. More precise specification of the mechanisms 
involved in the determination of $P_{L}(n)$ and $P_{F}(n)$ is required to generate unambiguous results.

For illustrative purposes, consider the following example. Rewards are completely nonappropriable; $P_{L}=P_{F}$. Furthermore, consider the simple linear demand-zero marginal cost oligopoly game where each firm captures $P_{F}=$ $4 P /(n+1)^{2}$, where $P$ is the value of a patent on the innovation, Then

$$
u_{i}^{*}\left(t, z ; P_{F}, P_{F}\right)=\frac{2 P_{F} \lambda e^{r t}}{2-(2 n-1) P_{F} \lambda^{2}\left(e^{r t}-e^{r T}\right) / r} .
$$

Now

$$
\partial u_{i}^{*} / \partial n=\frac{2 P_{F} \lambda e^{r t}(2 \hat{m})}{[2-(2 n-1) \hat{m}]^{2}} \leqq 0
$$

for all $t \leqq T$, where $\hat{m}(t)=P_{F} \lambda^{2}\left(e^{r t}-e^{r T}\right) / r$. Since

$$
\partial u_{i}^{*} / \partial P_{F}=\frac{4 \lambda e^{r t}}{[2-(2 n-1) \hat{m}]^{2}}>0
$$

and $d P_{F} / d n=-8 P /(n+1)^{3}<0$, it is clear that for this simple case $d u_{i}^{*} / d n$ is negative for all $t \leqq T$.

Thus we can easily construct reasonable examples which reverse the results of Proposition 6 when imitators are able to appropriate some of the innovator's (potential) reward.

\section{EQUILIBRIUM WITH ENTRY}

Dasgupta and Stiglitz $[2,3]$ use a Loury-type model to determine equilibrium market structure for the case of perfect patent protection. In the context of this model, entry will occur until the number of rivals is $n_{0}$ such that $J^{i}\left(u_{1}^{*}, \ldots, u_{n_{0}}^{*}\right)$ $=0$. Since

$$
J^{i}\left(u_{1}^{*}, \ldots, u_{n}^{*}\right)=P-\frac{2 P(n-1)}{2 n-1-\exp \left\{P \lambda^{2}(n-1)\left(1-e^{r T}\right) / r\right\}}>0
$$

for all $n$, the equilibrium market structure requires that $n_{0}=\infty$. The resulting Nash equilibrium strategies are

$$
u_{i}^{c}(t, z)=\lim _{n \rightarrow \infty} u_{i}^{*}(t, z ; P, 0)=P \lambda e^{r t} .
$$

These are the "competitive equilibrium" strategies using the definition of competitive equilibrium as the limit of the $n$-firm Nash equilibrium as $n \rightarrow \infty$.

Kamien and Schwartz [5] have argued that perfect patent protection is inconsistent with perfect competition. Instead, they characterize "perfect compe- tition" as immediate imitation by infinitely many rivals so that each firm "reaps a miniscule portion of the benefits of the innovation" (p. 56).

In terms of the present model, this definition of perfect competition requires that $P_{L}(n)$ and $P_{F}(n)$ both approach zero as $n$ approaches $\infty$. Then we see that the "perfectly competitive" rate of knowledge acquisition, when innovators and imitators are equally rewarded in the amount $P_{F}(n)$, is

$$
u_{i}^{p c}(t, z)=\lim _{n \rightarrow \infty} \frac{2 P_{F}(n) \lambda e^{r t}}{2-(2 n-1) P_{F}(n) \lambda^{2}\left(e^{r t}-e^{r T}\right) / r}=0
$$

for all $t \in[0, T]$.

With perfect competition defined as above, the results of this model coincide with those of Kamien and Schwartz [5] - technical advance is not a worthwhile undertaking for any individual firm.

From this it is clear that the extent of appropriability of rewards is a critical determinant of competitive behavior.

\section{SUMMARY AND CONCLUSIONS}

We have developed a model of optimal resource allocation to $\mathrm{R}$ and $\mathrm{D}$ under the assumption of uncertain technical advance and in the presence of gameplaying rivals. We have found that the availability of perfect patent protection unambiguously increases the pace of technical advance. For the case of perfect patent protection, we have seen that increasing the number of rivals results in an increase in each individual firm's Nash equilibrium rate of investment in $\mathbf{R}$ and D. When imitators are able to appropriate some of the benefits, then a greater number of rivals has several conflicting effects, affecting not only the probability of winning, but the values of the payoffs to innovator and imitators as well. Depending upon the payoff structure, increased rivalry in this case may accelerate or delay innovation.

\section{California Institute of Technology}

Manuscript received August, 1979; revision received May, 1981.

\section{REFERENCES}

[1] Bernstein, Dorothy L.: Existence Theorems in Partial Differential Equations. Princeton, New Jersey: Princeton University Press, 1950.

[2] Dasgupta, Partha, and Joseph Stiglitz: "Industrial Structure and the Nature of Innovative Activity," The Economic Journal, 90(1980), 266-293.

[3] - : "Uncertainty, Industrial Structure and the Speed of R and D," Bell Journal of Economics, 11(1980), 1-28.

[4] Friedman, Avner: Differential Games. New York: John Wiley and Sons, 1971.

[5] Kamien, Morton I., and Nancy L. Schwartz: "Timing of Innovations Under Rivalry," Econometrica, 40(1972), 43-60. 

[6] - $267-277$.

7] : "On the Degree of Rivalry for Maximum Innovative Activity," Quarterly Journal of Economics, 90(1976), 245-260.

[8] LeE, TOM, and Louis L. Wilde: "Market Structure and Innovation: A Reformulation," Quarterly Journal of Economics, 94(1980), 429-436.

[9] Loury, GLENN C.: “Market Structure and Innovation," Quarterly Journal of Economics, 93(1979), 395-410

[10] Reinganum, Jennifer F.: "Dynamic Games of Innovation," Journal of Economic Theory, $25(1981), 21-41$.

[11] Scherer, Frederic M.: "Research and Development Resource Allocation Under Rivalry," Quarterly Journal of Economics, 81(1967), 359-394.

[12] Stalford, H., and G. Leitmann: "Sufficiency Conditions for Nash Equilibrium in $N$-Person Differential Games," in Topics in Differential Games, edited by Austin Blaquiere. Amsterdam:

North-Holland Publishing Co., 1973. 